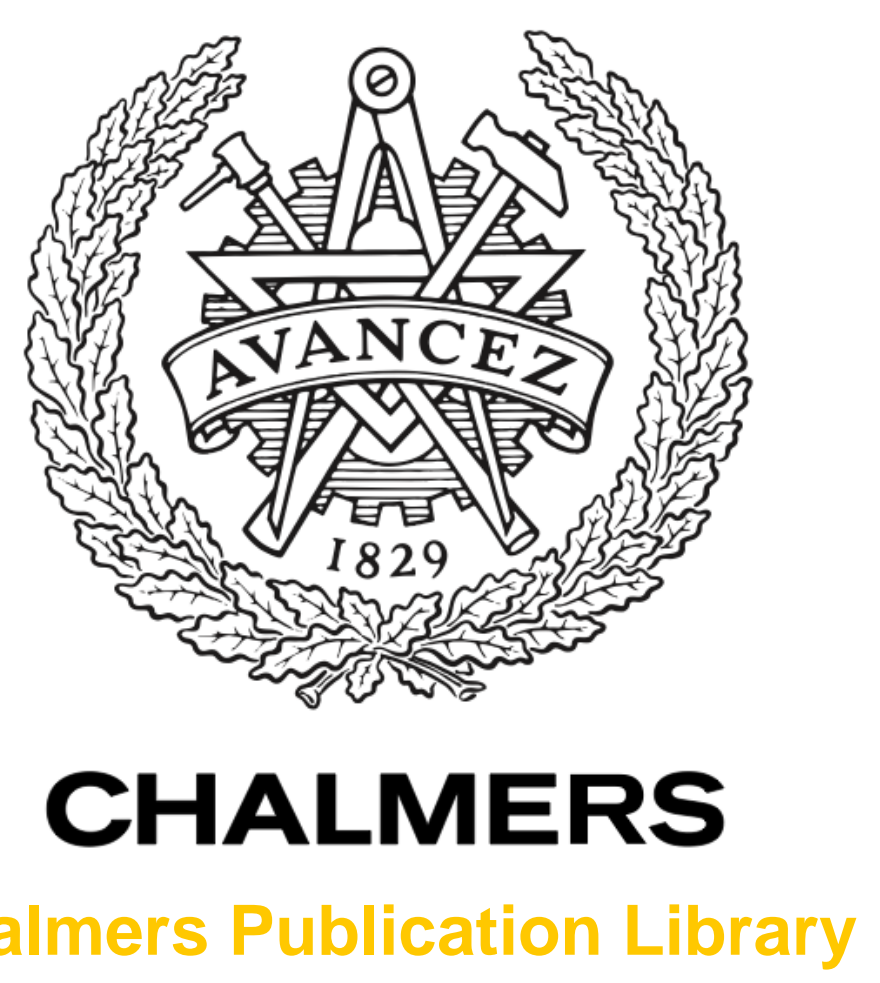

Challmers Publication Library

Modelling of Particulate Matter Transformations and Capture Efficiency

This document has been downloaded from Chalmers Publication Library (CPL). It is the author's version of a work that was accepted for publication in:

Canadian Journal of Chemical Engineering (ISSN: 0008-4034)

Citation for the published paper:

Sjöblom, J. ; Kannan, A. ; Ojagh, H. et al. (2014) "Modelling of Particulate Matter

Transformations and Capture Efficiency". Canadian Journal of Chemical Engineering, vol.

92(9), pp. 1542-1551.

http://dx.doi.org/10.1002/cjce.22004

Downloaded from: http://publications.lib.chalmers.se/publication/203366

Notice: Changes introduced as a result of publishing processes such as copy-editing and formatting may not be reflected in this document. For a definitive version of this work, please refer to the published source. Please note that access to the published version might require a subscription.

Chalmers Publication Library (CPL) offers the possibility of retrieving research publications produced at Chalmers University of Technology. It covers all types of publications: articles, dissertations, licentiate theses, masters theses, conference papers, reports etc. Since 2006 it is the official tool for Chalmers official publication statistics. To ensure that Chalmers research results are disseminated as widely as possible, an Open Access Policy has been adopted.

The CPL service is administrated and maintained by Chalmers Library. 


\title{
Modeling of particulate matter transformations and capture efficiency
}

\author{
Jonas Sjöblom, Ananda Subramani Kannan, Houman Ojagh and Henrik Ström* \\ Department of Applied Mechanics \\ Chalmers University of technology \\ SE 41296 Gothenburg \\ *Corresponding author, Email: henrik.strom@chalmers.se, Tel.: +46 (0) 317721360
}

\begin{abstract}
In the current work, a comprehensive computational fluid dynamics (CFD) model is developed for an accurate description of the transport and transformation of automotive particulate matter in monolithic reactors. The model accounts for the developing gas flow, the evaporation of hydrocarbons from the particles, the adsorption of hydrocarbons in the washcoat, as well as motion, shrinkage and deposition of particles in the channel. The comprehensive CFD model is used to validate a simplified tanks-in-series approach with a conceptual model for particulate matter transformations. In the development of more detailed and accurate chemical kinetics for the reactions of particulate matter in filters, it will be necessary to also predict the time-resolved properties of the particles collected in the filter (i.e. reactivity, amount of adsorbed hydrocarbons, etc.). It is shown in this work how the data necessary to construct such models can be obtained in-situ with the aid of the conceptual model and particulate matter measurements over an inert open substrate.
\end{abstract}

Keywords: particulate matter, deposition, evaporation, transformation, soot

\section{Introduction}

Non-ideal combustion in automotive internal combustion engines results in the formation of particulate matter (PM). PM in the atmosphere has a significant impact on both global warming and ozone depletion [1], as well as detrimental effects on human health.[2] The most common way of reducing the PM content of the exhaust gases in heavy-duty applications is by forcing the gas through a wall-through filter [3], where particles are deposited at the cost of a significant imposed pressure drop.

Open filters (metallic or ceramic monolithic flow-through reactors with low pressure drop) have potential for energy efficient reduction of PM from internal combustion engines and, for some applications, may even suffice. The successful development, design and operation of such substrates are dependent on the availability of adequate mathematical models. However, the PM from internal combustion engines is prone to changes (via changes in gas composition and temperature) and it is therefore very challenging to measure and characterize.[4-12] The modeling of these systems is also challenging due to the variability in size-dependent heterogeneous properties.[13-17] In addition, it is well established experimentally that the passage of PM through an open substrate may significantly alter both the size distribution as well as the chemical composition of the PM.[18-20] Hence, there is a need for a comprehensive model that takes into account not only the transport of PM in open substrates, but also the PM transformations that occurs inside the channels. The aim of this work is to derive, validate and showcase such a model. 
Recently, the capture efficiency of PM in open substrates (bare cordierite and alumina-coated cordierite monoliths) was investigated using PM from a real engine under various flow conditions (varying residence times and temperatures) and sampling settings (dilution ratios).[21] However, the capture efficiency was affected by PM transformations in terms of a removal of volatiles (hydrocarbons, HCs) that influences both the particle sizes and numbers. In order to quantify these effects, a conceptual model was proposed. This conceptual model has the potential to be used as an insitu analyzer of the PM properties, once it has been calibrated.

In this paper, a detailed derivation of the conceptual model for PM transformations is presented (Section 2) and it is shown how the model parameters are obtained from experiments. Thereafter, a comprehensive computational fluid dynamics (CFD) model of the flow of PM and exhaust, including PM-exhaust interactions and PM transformations, is constructed (Section 3). CFD simulations that validate the assumptions made in the derivation of the conceptual model are presented in Section 4, together with an assessment of the influence of the PM transformations under real engine conditions. The paper finally concludes with a summary of the findings and their implications for PM characterization measurements, including how the model can be used for in-situ analysis of the PM properties in full scale experimentation.

\section{A conceptual model for PM transformations}

PM is generally considered a heterogeneous material with a pronounced $\mathrm{HC}$ content, especially in the smaller particles. Consequently, HC evaporation may affect the particle size distribution of the PM passing through a monolith substrate in two different ways. First, there is a possibility that particles consisting only of HCs evaporate completely inside the channel, leading to an apparent increase in the particle trapping efficiency. Second, incomplete evaporation of $\mathrm{HCs}$, or complete evaporation of $\mathrm{HCs}$ from particles that have a solid core underneath the condensed HCs, will reduce the size of the affected particles, leading to an enhanced diffusivity and hence a higher trapping efficiency.

\subsection{Particle types}

To account for the effects of these two pathways to PM transformation and their subsequent effect on the particle size distribution, a conceptual model is introduced. In this model, the particles are classified into three groups:

- Group I: Soot/solid particles without any condensed HCs

- Group II: Particles with condensed HCs over the surface and (non-volatile) soot inside

- Group III: Droplets consisting entirely of condensed HCs

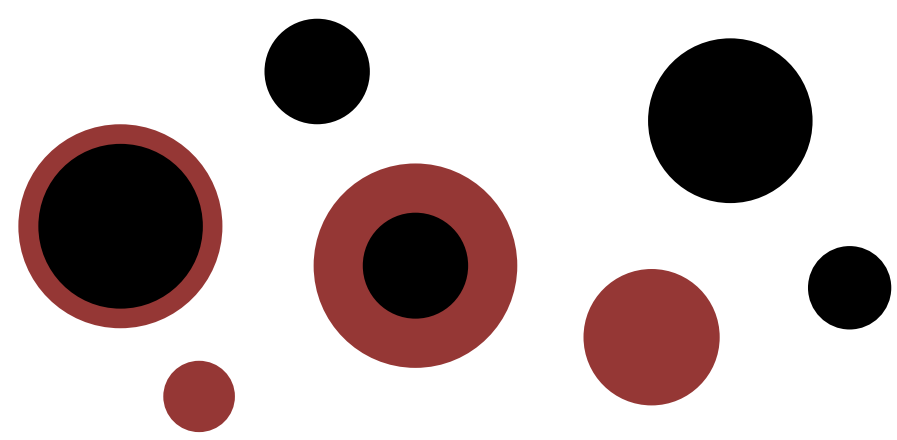

Figure 1: A conceptual model for the PM used for capture experiments. The black color represents non-volatile component (soot) and the brown color represents the volatile component $(H C)$. 
The model makes use of two parameters, $\mathrm{HC}_{\mathrm{part}}$ and $\mathrm{HC}_{\text {frac }}$, where the former is the mass fraction of pure HC particles (group III) and the latter is the mass fraction of HCs in the remaining particles. Both parameters are assumed to be functions of the particle size. Hence, the fraction of group III particles and the distribution between group I and group II particles among the remainder are allowed to vary with the particle size. The actual dependence of $\mathrm{HC}_{\text {part }}$ and $\mathrm{HC}_{\text {frac }}$ on the particle size for a certain particle size distribution is however unknown and must be determined experimentally by fitting parameters e.g. to a sigmoid function.

The core principle of the conceptual model is that $\mathrm{HCs}$ in the gas phase that enters the monolith channel will adsorb in the washcoat, which acts as a HC sink/trap. This will lower the bulk concentrations of HCs, which will start to drive off HCs from the particles. To test the validity of this assumption under the experimental conditions for which the conceptual model was derived, it becomes necessary to account for the simultaneous evaporation, convective and diffusive transport and adsorption of $\mathrm{HCs}$, together with the particle motion and deposition. This is precisely the aim of the CFD model derived later in Section 3.

In essence, the conceptual model constitutes a classification of the PM into three groups. The distribution of particles among these groups for a given particle size distribution (PSD) can be found by fitting the parameters in a postulated expression for the variables $\mathrm{HC}_{\text {part }}$ and $\mathrm{HC}_{\text {frac }}$ (e.g. eq. (7)) so that the apparent particle capture efficiency observed experimentally can be reproduced by a tanks-inseries model. When the dependence of these two variables on the particle size is known, numerical predictions of the PM transformations becomes possible in any numerical framework of choice (i.e. tanks-in-series models, as well as comprehensive CFD models and efficient reduced on-line models).

Next, we introduce the experimental procedures used to obtain the PSDs before and after an open filter. Thereafter, we present the implementation of the conceptual model for PM transformations into a tanks-in-series model for PM deposition in a monolith channel. This model is used to fit the parameters in the expressions for $\mathrm{HC}_{\text {part }}$ and $\mathrm{HC}_{\text {frac }}$. In the tanks-in-series approach, it becomes necessary to make a number of assumptions regarding the gas phase flow, temperature and concentration fields. Consequently, we finally derive a comprehensive CFD model that resolves the actual motion and transformation of PM in the developing gas flow, simultaneously accounting for the evaporation of hydrocarbons from the particles, the adsorption of hydrocarbons in the washcoat, and the motion, shrinkage and deposition of particles in the channel. The CFD model is used to critically assess the assumptions inherent in the parameter fitting procedure with the tanks-in-series model and to estimate the effects of PM transformations on the apparent PM capture efficiency under various other conditions where experimental data is not available.

\subsection{Experimental setup}

The experiments were performed using a diesel engine (Volvo D5) connected to an Exhaust AfterTreatment System (EATS), as shown in Figure 2. The experimental setup has been described in detail in $[21,22]$ where temperature and flow (channel residence times) were systematically varied together with variations in the sampling conditions to verify the findings. 


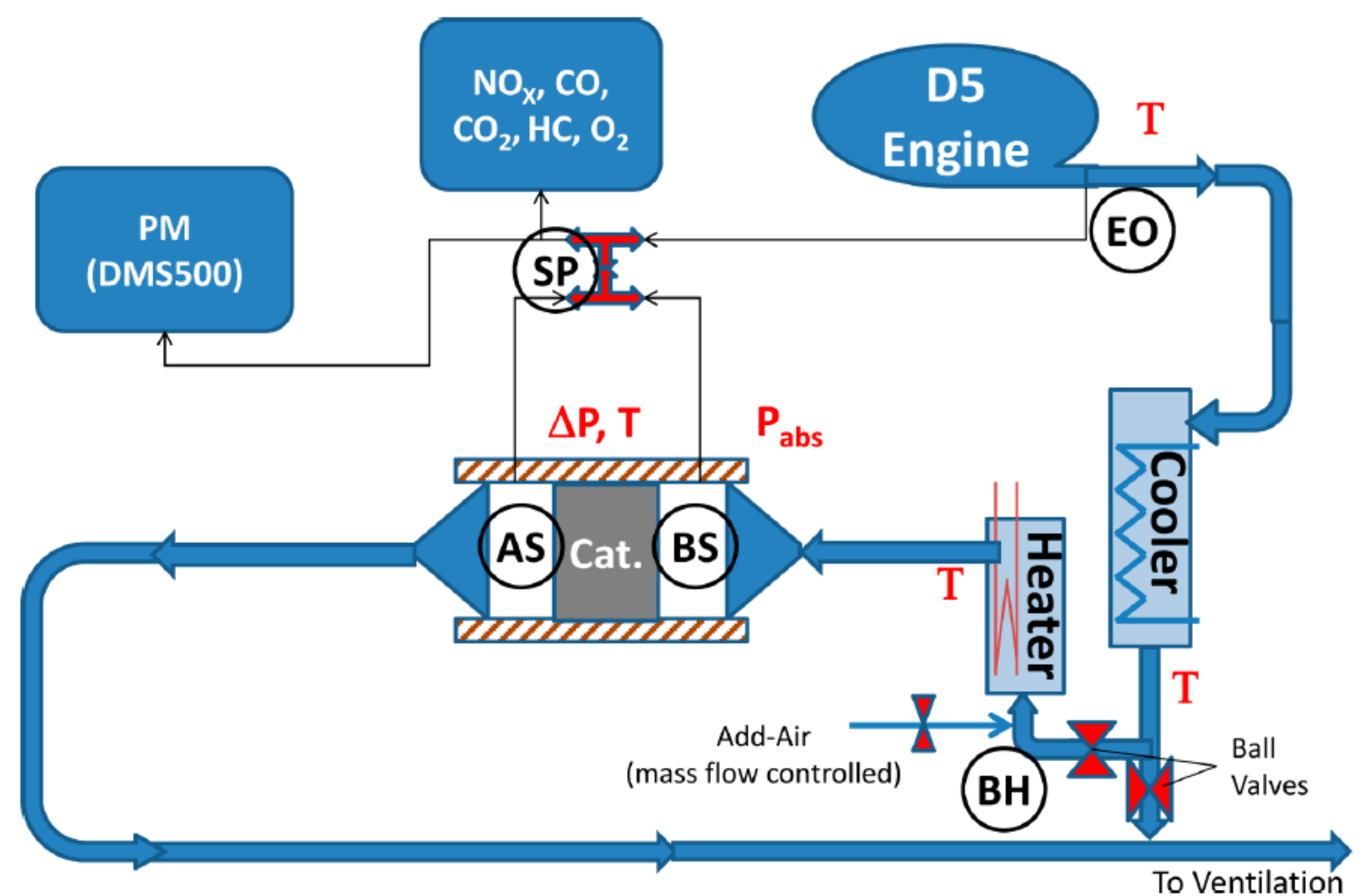

Figure 2: The engine and the Exhaust AfterTreatment System (EATS) setup. Legend: EO, engine out; $B H$, before heater; BS, before substrate; AS, after substrate; and SP, switch point.

In the current work, one experimental data set is chosen for further investigation (the set labelled "Case B4" in [21]). The motivation to using this particular data set stems from the fact that it features both high trapping efficiencies (strong data signals) as well as significant deviations from existing theoretical predictions (strong potential HC-effects). A detailed validation test for a single data set is deemed sufficient, since it has already been shown [21] that the magnitude and direction of the deviations investigated here could be explained within the experimental uncertainty by a mathematical model that predicts PM transformations in conjunction with the transport and deposition. Furthermore, an additional simulation under real-engine conditions will also be performed to assess the possible influence of PM transformations under more realistic operation conditions.

In the chosen experiment, a monolith coated with bare alumina was used and the engine was run at $1800 \mathrm{rpm}$ and $80 \mathrm{Nm}$. There was no addition of air to the exhaust and the temperature was kept at $154^{\circ} \mathrm{C}$. The flow was $39 \mathrm{slpm}$ corresponding to a channel Reynolds number ${ }^{1}$ of 2.0 and a channel residence time of $2.95 \mathrm{~s}$. The PM was measured using a DMS500 (fast particulate spectrometer from Cambustion) using a primary dilution factor of 4 and a secondary dilution factor (rotating disc dilution) of 16. The PM losses due to deposition in the sampling pipes and to the dilution in the DMS500 were compensated for, so that all PSDs used are independent of such experimental influences.[21] The measured (apparent) size-resolved particle capture efficiency (CE) is defined as:

$C E=\frac{P S D_{\text {before }}-P S D_{\text {after }}}{P S D_{\text {before }}}$

The PSD measured by the DMS500 generated 38 signals (from 4 to $1000 \mathrm{~nm}$ ), where the signal was different from zero for approximately 20 size bins in the present experiment. From the analysis of many measurements, the uncertainty could also be estimated.[21]

\footnotetext{
1 The channel Reynolds number is based on the hydraulic diameter of the channel and the average linear channel velocity (at actual conditions).
} 


\subsection{Tanks-in-series setup}

The capture efficiency is modeled as a single channel model, discretized using a tanks-in-series approach with 10 tanks. In each tank, the evaporation is modeled using the approach from Giechaskiel and Drossinos [23] with material data for tetracontane (C40, properties as given in [23]):

$$
\frac{d\left(d_{p}\right)}{d t}=\frac{2 M_{H C} a_{c}\left(p_{\infty}-p_{d}\right)}{\rho_{p} N_{A} \sqrt{2 \pi m_{H C} k_{B} T}}
$$

Here, the change in particle diameter that results from the evaporation of surface HCs is calculated from the molar flux at the surface and with the rarefied conditions around the small particles taken into account. One important assumption made in our previous study was that the bulk concentration (expressed as $p_{\infty}$ ) was assumed to be zero, whereas this assumption will be relaxed in the current investigation.

To control the shrinking process, the residence time in each tank was further discretized in 100 time steps. After the calculation of the shrinking process, the diffusion losses were calculated according to Brownian deposition theory [24]:

$$
C E_{\text {channel }}=\left(1-P E_{\text {channel }}\right)=\exp \left(\frac{-h_{m} A_{s}}{q}\right)
$$

In other words, the shrinkage was treated as being decoupled from the particle motion. The average mass transfer coefficient, $h_{m}$, is obtained via the Sherwood number:

$$
h_{m}=\frac{D_{p} S h}{d_{c h}}
$$

Here, $S h$ is the Sherwood number based on the channel diameter. Although the approach represented by eq. (3-4) is general, it relies on the availability of an adequate Sherwood number correlation. The Sherwood number correlation used in this work is derived from numerical solutions to the corresponding heat transfer problem [25] where the "local Sh" (in contrast to a channel averaged $S h$ ) is used:

$$
S h=N u_{\infty, T}+8.827\left(1000 z^{*}\right)^{-0.545} \exp \left(-48.2 z^{*}\right)
$$

where the asymptotic Nusselt number, $N u_{\infty, T}$, depends on the channel geometry (in this case $N u_{\propto, T}=$ 2.976) and $\mathrm{z}^{*}$ is a dimensionless axial coordinate:

$$
z^{*}=\frac{z D_{p}}{u d_{c h}^{2}}
$$

At the outlet of the channel, the particles belonging to group II have sizes that do not match the sizes from the instrument. The penetration efficiency was therefore used when interpolating to the size bins of the instrument. 
The inlet PSD can be divided into three groups: pure HC (group III) and semivolatile PM (group II). One part $\left(\mathrm{HC}_{\mathrm{part}}\right)$ belongs to group III and the rest belong to group II. Group II also features a subgroup (group I) that contains only pure soot particles. The semivolatile part of the PSD is assigned a certain mass fraction of $\mathrm{HC}\left(\mathrm{HC}_{\text {frac }}\right)$ that covers the outer surface of the PM. The relative amount of $\mathrm{HC}_{\text {part }}$ and $\mathrm{HC}_{\text {frac }}$ need to be size-dependent and smaller PM can contain more $\mathrm{HC}$ than larger PM. In order to make an analytical expression for these properties, a sigmoidal function was chosen. Two functions (generalised logistic function, adapted from [26]) were applied, both in the equation form:

$$
H C_{\text {distributon }}=A+\frac{(K-A)}{\left(1+Q e^{-B(t-M)}\right)^{1 / v}}
$$

In the equation above, the parameters $A$ and $K$ are the asymptotic values at smaller and larger particles respectively, $Q$ and $v$ represent the "rounding" near the asymptotes, $B$ is the maximum slope, $M$ the position of the inflexion point (if $Q=v$ ) and $t$ is the dependent variable, in this case the logarithm of the particle size (in $\mathrm{nm}), \log _{10}\left(d_{p}\right)$.

This function has the properties of being physically sound, being a smooth function and flexible enough to fit to the experimental data. Note that the real PM is far more complex that this conceptual model (having non-spherical shape, having a variety of $\mathrm{HC}$ content, etc). However, the model should be very useful since it can be used as an in-situ analyzer of the $\mathrm{HC}$ characteristics, which is valuable when studying complex phenomena such as the present ones.

\subsection{Parameter estimation results}

A total of 8 parameters (shown in Table 1) were adjusted by applying a gradient search function (lsqnonlin) in Matlab. The parameter correlation was very high (different sets of parameters could give very similar results, especially the balance between $\mathrm{HC}_{\text {part }}$ and $\mathrm{HC}_{\text {frac }}$ ) so confidence intervals are not reported. It should be stressed here that the parameters values reported in Table 1 are specific to the chosen experimental data set and that the parameters need to be refitted to relevant experimental data for use under different operating conditions.

Table 1: Tuned parameter values for $H C_{\text {part }}$ and $H C_{\text {frac }}$ in eq. (7).

\begin{tabular}{|l|c|c|}
\hline & $\mathbf{H C}_{\text {part }}$ & $\mathbf{H C}_{\text {frac }}$ \\
\hline$A$ & 0.744 & 0.393 \\
\hline$K$ & 0.000 & 0.000 \\
\hline$Q$ and $v$ & 0.343 & 0.343 \\
\hline$B$ & 7.469 & 10.148 \\
\hline$M$ & 1.738 & 1.728 \\
\hline
\end{tabular}

The results from the parameter estimation for the experimental data set used are displayed in Figure 3. The physical interpretation of the fitted distribution functions is that a significant part of the smaller PM consists of pure HCs, and that semivolatile particles are present (to a quite low extent) as intermediate sized PM in the approximate range $25-60 \mathrm{~nm}$. There is no predicted $\mathrm{HC}$ contribution to particles larger than $90 \mathrm{~nm}$. 


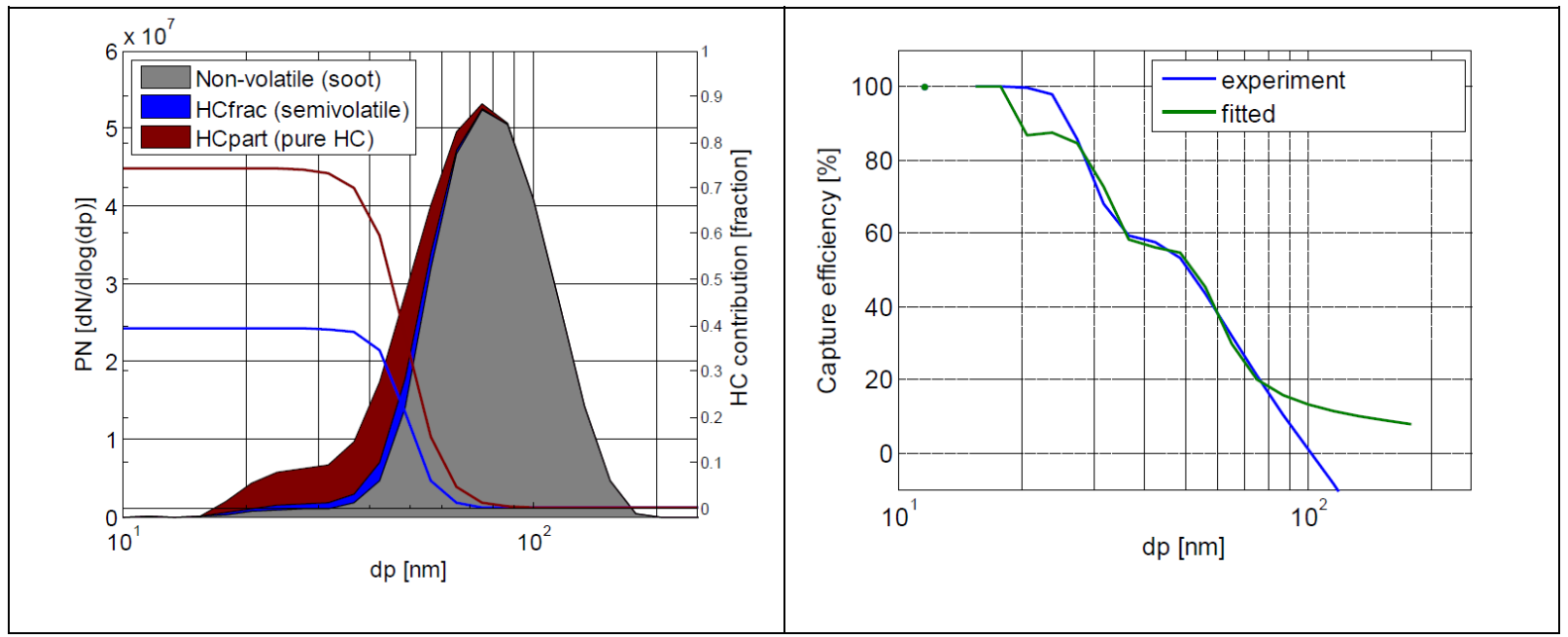

Figure 3: PSD and the fitted HC contribution (left) necessary to explain the experimental capture efficiency (right) for the alumina substrate.

\section{CFD modeling}

The conceptual model, implemented into a tanks-in-series setup, and with the parameter set reported in Table 1, is to be compared with more detailed numerical simulations using the CFD approach introduced in this section.

The simultaneous motion and deposition of, and possible $\mathrm{HC}$ evaporation from, the particulate matter is most straightforwardly simulated in a finite-volume framework using the Eulerian-Lagrangian approach. With this technique, balance equations for the continuous (gaseous) phase are solved numerically in an Eulerian frame of reference, whereas the dispersed phase is treated in a Lagrangian frame of reference by the tracking of a large number of computational parcels that represent different types of particles. The computational domain is $1 / 4^{\text {th }}$ of one monolith channel (i.e. including the full length of the channel with two symmetry planes in the wall-normal directions). The following assumptions are made:

- The fluid flow is incompressible.

- The influence on the velocity field from the changes in temperature or HC concentration and from the momentum transfer with the particulate phase is negligible, and hence the developing velocity field from the channel inlet throughout the channel can be kept frozen during the tracking of the particles. This is a good assumption, as the HC concentration levels and the particulate loading are both low and the expected temperature changes are very minor.

- Heat transfer due to species diffusion and viscous dissipation is neglected.

- Mass transfer due to thermal effects is neglected.

- The HC content in the particles and in the gas phase consists solely of tetracontane. Tetracontane is expected to be one of the heaviest HC components present, and this assumption thus leads to an underestimation of the rate of evaporation of HCs from the particles. If the conceptual model can be validated using this assumption, it will be valid for all other HCs.

- The partial pressure of tetracontane at the channel inlet is equal to the saturation vapor pressure of tetracontane at the current pressure and temperature. Because of the presence of very small particles/droplets, this assumption results in an overprediction of the amount of HC that has to be adsorbed at the channel walls before the evaporation of $\mathrm{HC}$ from the particles begins. Hence, this assumption is in line with the aim to put the validity of the conceptual model to the test.

- The partial pressure of $\mathrm{HC}$ at the channel walls is zero, corresponding to total adsorption of $\mathrm{HC}$ in the washcoat. This assumption could (theoretically) be affected by the catalyst composition in the monolith, but such effects are most probably negligible during transient 
operation due to the dominance of the number of adsorption sites in the washcoat over the typical number of catalytically active sites. In the event of long term steady-state operation, saturation could become an issue and it is then possible for catalyzed reactions to become important. However, the experimental data used in the current work were obtained for inert alumina-coated substrates and thus bear no effect of catalyzed reactions.

The set of equations that define the mathematical model used for the channel simulations are introduced in the following. The gas phase balance equations (eqs. (9), (10) and (11)) equate the sum of the temporal change and the convective transport to the net contribution of the molecular effects (plus relevant source terms from the two-phase interaction). The ancillary equations needed are discussed in the respective subsections.

\subsection{Gas phase velocity and pressure fields}

The gas phase continuity equation and momentum balance equations are:

$\nabla \cdot u=0$

$\rho\left[\frac{\partial u}{\partial t}+u \cdot \nabla u\right]=-\nabla P+\mu \nabla^{2} u$

The material properties of the exhaust gases are taken to be those of air at the same pressure and temperature. The boundary conditions applied are: a uniform velocity at the channel inlet, zero velocity at the channel walls and zero gradients of pressure and velocity normal to the channel outlet and to the symmetry planes.

\subsection{Gas phase temperature field}

The gas phase temperature field is obtained from the gas phase energy balance equation:

$\frac{\partial}{\partial t}\left(\rho h_{E}+\frac{\rho u^{2}}{2}\right)+\nabla \cdot\left(u\left(\rho h_{E}+p+\frac{\rho u^{2}}{2}\right)\right)=\nabla \cdot(k \nabla T)+S_{H}$

Here, $h_{E}$ is the sensible enthalpy (defined as $h_{E}=\int_{T_{r e f}}^{T} c_{p} d T$ ) and $S_{H}$ is the source term from the Lagrangian particle tracking (defined in Section 3.5). The boundary conditions applied are: a uniform temperature at the channel inlet, adiabatic channel walls and zero temperature gradient normal to the channel outlet.

\subsection{Gas phase concentration fields}

The gas phase concentration field of tetracontane is obtained from the corresponding convectiondiffusion equation:

$\rho \frac{\partial Y_{H C}}{\partial t}+\nabla \cdot\left(\rho u Y_{H C}\right)=-\nabla \cdot\left(\rho D \nabla Y_{H C}\right)+S_{M}$

Here, $S_{M}$ is the source term from the Lagrangian particle tracking (defined in Section 3.6). The boundary conditions applied are: a uniform mass fraction at the channel inlet, zero mass fraction at the channel walls and zero gradient normal to the channel outlet.

\subsection{Particle motion and initialization}

The particles are accounted for by computational parcels that represent a number of real particles, $N_{P}$. Their trajectories are obtained from the following particle equations of motion: 
$\frac{d x_{p, i}}{d t}=u_{p, i}$

$m_{p} \frac{d u_{i}}{d t}=3 \pi \mu d_{p}\left(u_{i}-u_{p, i}\right) / C_{C}+\varsigma_{i} \sqrt{\frac{6 \pi \mu d_{p} k_{B} T}{C_{C}}}$

Eq. (12) relates the particle positions to their respective velocities, and eq. (13) is Newton's law of motion equating the product of the particle mass and its acceleration to the net force acting on the particle. Here, $\varsigma_{i}$ is a normally distributed random number of zero mean and unit variance. The two terms on the right hand side of eq. (13) represent the Cunningham-corrected Stokes drag and the Brownian motion, which are known to be the two most relevant forces acting on automotive particulate matter in the current size range.[27,28] Particles that reach the channel walls are assumed to deposit there, whereas the symmetry planes act as reflecting walls for the particle motion.

In the simulations, a number of computational parcels of $O(100)$ are introduced every time step, so that the total number of parcels in the channel when it is filled with particles is of $O\left(10^{4}\right)$. Consequently, $N_{P}$ is of $O(100)$. Upon injection, the parcel is assigned a random position on the plane that represents the channel inlet, and a velocity equal to that of the gas. The particle diameter is sampled from a cumulative particle size distribution function that is tuned to the experimental data for the validation case.[21] The sampling is accomplished using a uniformly distributed random number, $r_{1}$, on the interval $(0,1)$. The probability that a particle of diameter $d_{p}$ consists entirely of HCs is given by $\mathrm{HC}_{\text {part }}$ In accordance with the acceptance-rejection method, the particle is therefore initialized as a pure $\mathrm{HC}$ particle if $r_{2}<\mathrm{HC}_{\text {part, }}$, where $r_{2}$ is a new uniformly distributed random number on the interval $(0,1)$. If $r_{2} \geq \mathrm{HC}_{\text {part }}$, the particle is instead initialized to contain a fraction $\mathrm{HC}_{\text {frac }}$ of $\mathrm{HCs}$. All material properties, including the particle density, are taken as the linear combination of the respective value for tetracontane and for graphite, based on the current $\mathrm{HC}$ content in the particle.

It should be stressed here that whereas injection of particles using the actual PSD is the only acceptable method to assess the effects of the two-way coupled HC evaporation, it will necessarily mean that particle sizes close to the tails of the PSD employed are not present in high enough numbers to obtain statistical significance in terms of their capture efficiency. This implies that the CE values obtained for the smallest and largest particle sizes present should be interpreted cautiously.

\subsection{Particle temperature}

Changes to the particle temperature occur from the combined effect of evaporation and convective heat exchange with the gas phase. The particle temperature is therefore monitored via the following particle heat balance equation:

$m_{p} c_{p} \frac{d T_{p}}{d t}=h A_{p}\left(T-T_{p}\right)+\Delta H_{v a p} \frac{d m_{p}}{d t}$

The heat transfer coefficient, $h$, is obtained from the correlation of Mikami et al. [29], which accounts for the effects of rarefied flow around the particles. The current rate of evaporation $\left(d m_{p} / d t\right)$ is obtained from the particle mass balance (cf. Section 3.6). The initial temperature of the particles upon injection is equal to that of the gas phase. The source term in the continuous phase energy balance is obtained by summing, in each cell, over the contributions from each parcel, magnified by the number of real particles represented by the computational parcel:

$S_{H}=\sum_{j=1}^{N_{C}} N_{P, j} m_{p, j} c_{p, j} \frac{d T_{p, j}}{d t} / V_{C}$

In this formulation, $S_{H}$ is written on a per-cell basis and $N_{C}$ is the total number of parcels in the cell.

\subsection{Particle HC content}


If the particle (still) contains any $\mathrm{HCs}$, the particle mass loss due to evaporation is obtained from the following particle mass balance equation [23]:

$\frac{d m_{p}}{d t}=\frac{2 m_{H C} a_{c} A_{p}\left(p_{H C, \infty}-p_{H C, S}\right)}{\sqrt{2 \pi m_{H C} k_{B} T_{p}}}$

Eq. (16) is equivalent to eq. (2) but on a mass basis. Whenever the particle HC content is zero, $d m_{p} / d t$ is also set equal to zero. The study of local condensation phenomena, if present, is outside the scope of this work, and negative values of $d m_{p} / d t$ are also set equal to zero.

The partial pressure of tetracontane in the cell is obtained from the mass fraction $Y_{H C}$ using Raoult's law, and that at the particle surface is calculated via the Kelvin equation (accounting for the curvature of the droplet surface):

$p_{H C, s}=p_{H C, s a t} \exp \left[\frac{4 \gamma M_{H C}}{\rho_{H C} R T_{p} d_{p}}\right]$

During the initialization of the particle mass (cf. Section 3.4), an additional variable $\varphi$ that stores the current particle HC content is also created. This variable is updated together with eq. (16) and is used to monitor the fraction of $\mathrm{HCs}$ remaining in the particle at all times:

$\varphi=m_{H C} / m_{p}$

The source term in the continuous phase species transport equation is obtained by summing, in each cell, over the contributions from each parcel, magnified by the number of real particles represented by the computational parcel:

$S_{M}=\sum_{j=1}^{N_{C}} N_{P, j} \frac{d m_{p, j}}{d t} / V_{C}$

In this formulation, $S_{M}$ is written on a per-cell basis and $N_{C}$ is the total number of parcels in the cell.

\subsection{Numerical details}

The problem to be solved constitutes a system of coupled partial differential equations (eqs. (8-9), (10) and (11)) and ordinary differential equations (eqs. (12-13), (14) and (16)). The solution procedure is iterative, with the source terms in the continuous phase balance equations being updated after every continuous phase time step and the particle momentum, heat and mass balance equations being solved in a coupled manner with the continuous phase balance equations.

The computational results are obtained using the commercial CFD software ANSYS Fluent 13.0. The third-order accurate, bounded QUICK scheme is used with very fine, structured hexahedral meshes for the discretization of the convective terms in the momentum, energy and mass balance equations. The diffusion terms are discretized using a second-order accurate central differencing scheme. The pressure-velocity coupling is handled using the SIMPLE scheme, and the pressure interpolation scheme uses the momentum equation coefficients.[30] The temporal discretization is first-order implicit for all continuous phase balance equations, and the time step is small to maintain stability in the two-way coupling.

In accordance with the theoretical requirements for the solution of eq. (14), the time step used in the integration of the particle equation of motion is always smaller than the particle relaxation time.[31] Again, this also works so as to enhance the numerical stability of the solution procedure of the coupled system. The temporal discretization of the particle balance equations is first-order explicit, which is consistent with the correct interpretation of the stochastic integral resulting from eq. (14).[32] 


\section{Results and Discussion}

\subsection{Model verification}

The numerical implementation of the conceptual model is tested by performing a CFD simulation of a case with only soot particles and comparing the results (in Figure 4) to the previously validated approach of Ström et al.[27] It is concluded that the agreement is satisfactory and the current implementation is therefore deemed verified. Again, it should be noted that the current approach does not produce any results for particle sizes outside the range of the herein employed PSD.

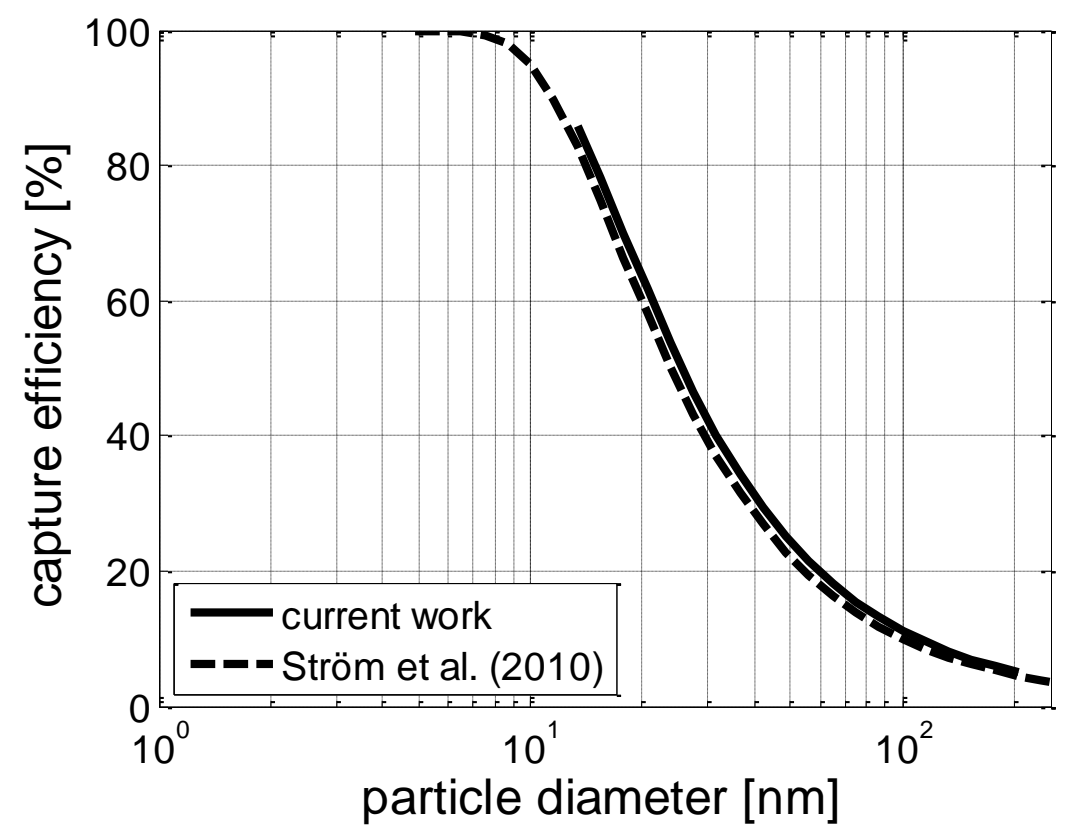

Figure 4: Capture efficiency of pure soot particles versus particle diameter for the CFD setup of the current work in comparison to the previous numerical model of Ström et al. [27].

\subsection{Validation test of the conceptual model}

The conceptual model is tested in a validation simulation. Figure 5 illustrates the capture efficiency in a representative monolith channel as a function of the particle size. This apparent capture efficiency is calculated from the differences in the particle size distributions at the channel inlet and outlet, and hence includes the effects of PM transformations and evaporation. As a result, this capture efficiency is significantly higher than that assuming inert PM (i.e. pure soot), as can be seen from the comparison with the results from Section 4.1. The agreement with the predictions from the tanks-in-series implementation of the conceptual model is satisfactory, given the numerical differences observed in Figure 4 and the additional uncertainty introduced by the use of computational parcels in the Lagrangian particle tracking approach (cf. Section 3.4). 


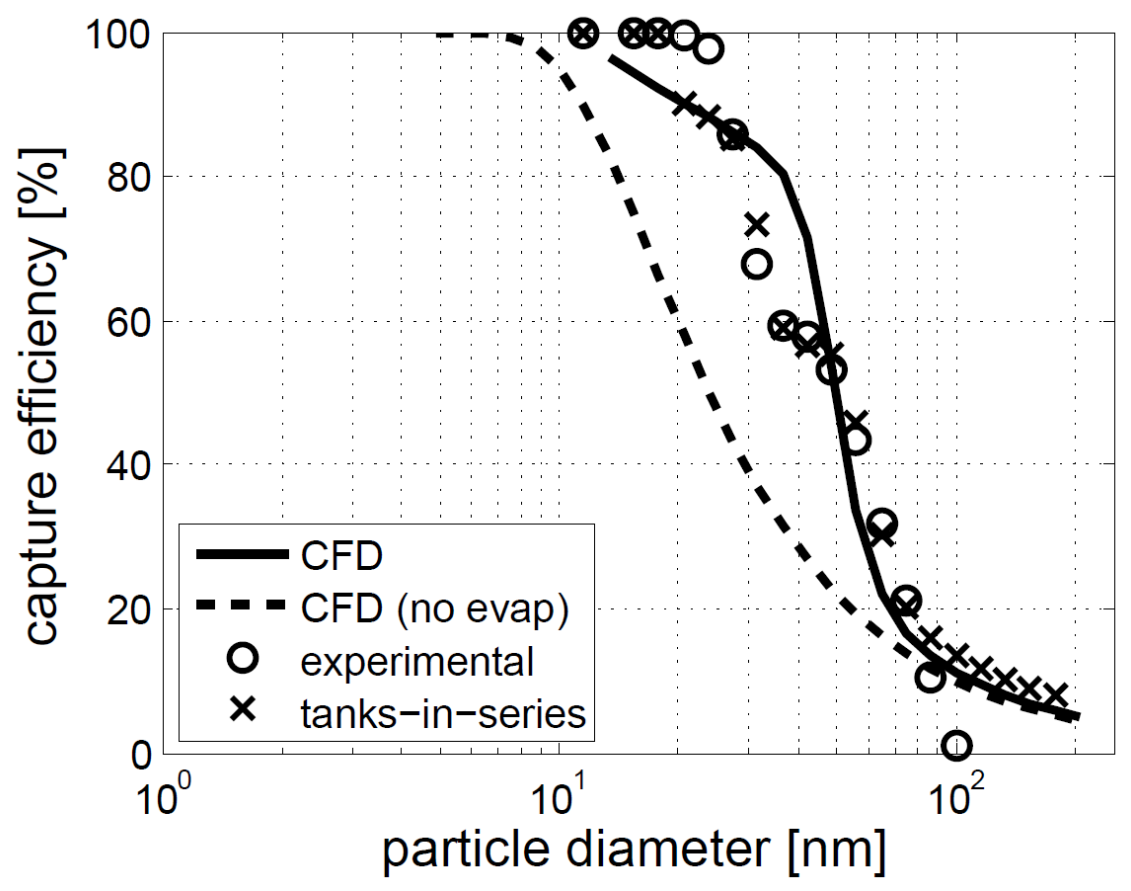

Figure 5: Apparent capture efficiency in the validation simulation. Also plotted is the capture efficiency due to wall deposition only (no evaporation) of the untransformed injected PSD, as well as the experimental data and an indication of the prediction from the tanks-in-series implementation of the conceptual model.[21]

The results obtained from Figure 5 hence indicate that the previous assumption that the HC evaporated from the particles in the channel will adsorb on the washcoat almost instantaneously is acceptable. It is the efficient transport of even a heavy $\mathrm{HC}$ such as tetracontane from the gas bulk to the monolith walls that makes possible a pronounced evaporation of HC particles as well as an increase in the wall deposition due to the associated particle shrinkage. The background levels of HCs present in the channel as predicted by the current method are investigated and found to be very low after a short initial distance, which confirms the conclusions from our previous work.[21] The quick disappearance of the HC-containing particles is also clearly visible in Figure 6. 


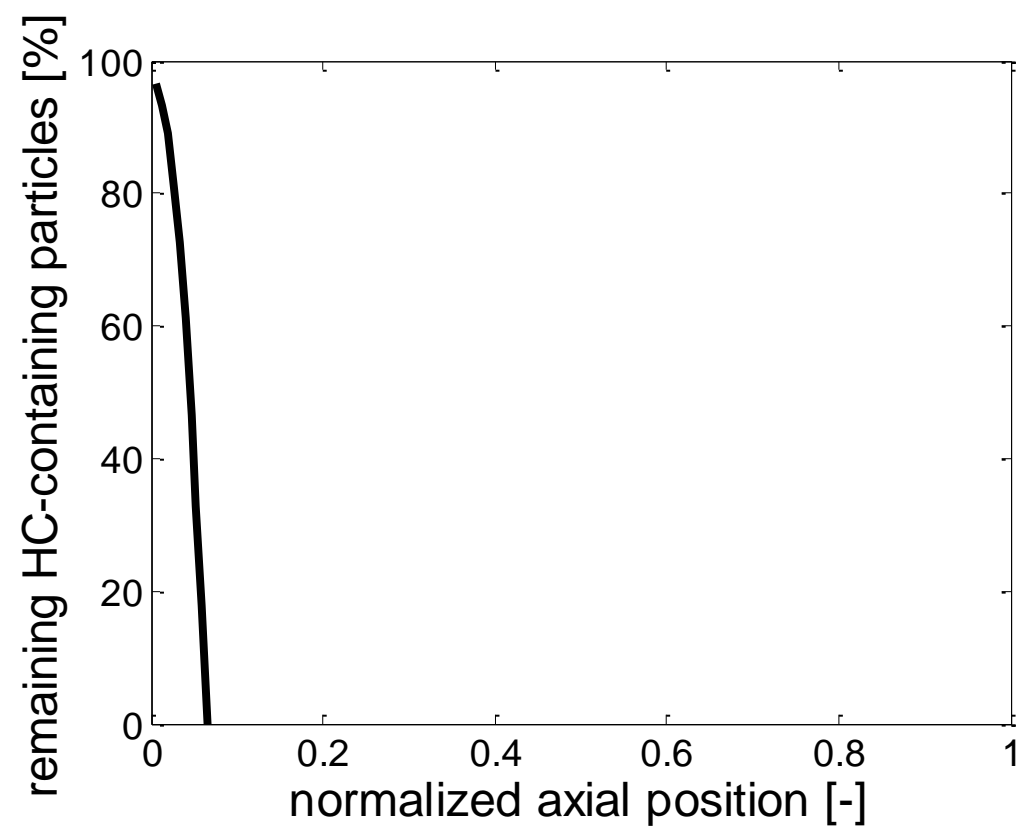

Figure 6: Number percentage of HC-containing particles present in the channel as a function of the axial distance into the monolith channel for the validation simulation.

HC evaporation may influence the apparent capture efficiency not only by complete HC evaporation. There can be a pronounced effect on the particle deposition rates from particle shrinkage caused by $\mathrm{HC}$ evaporation, as may be appreciated by plotting the particle diffusivity as a function of the particle size (Figure 7). There is an increase in the particle diffusivity of more than three orders of magnitude when going from $200 \mathrm{~nm}$ down to $5 \mathrm{~nm}$. When a particle has shrunk down to about one nanometer in diameter, its diffusivity is indeed similar to that of a gas phase molecule. Consequently, PM transformation via $\mathrm{HC}$ evaporation may increase the apparent capture efficiency either by this process or by complete evaporation of pure $\mathrm{HC}$ particles.

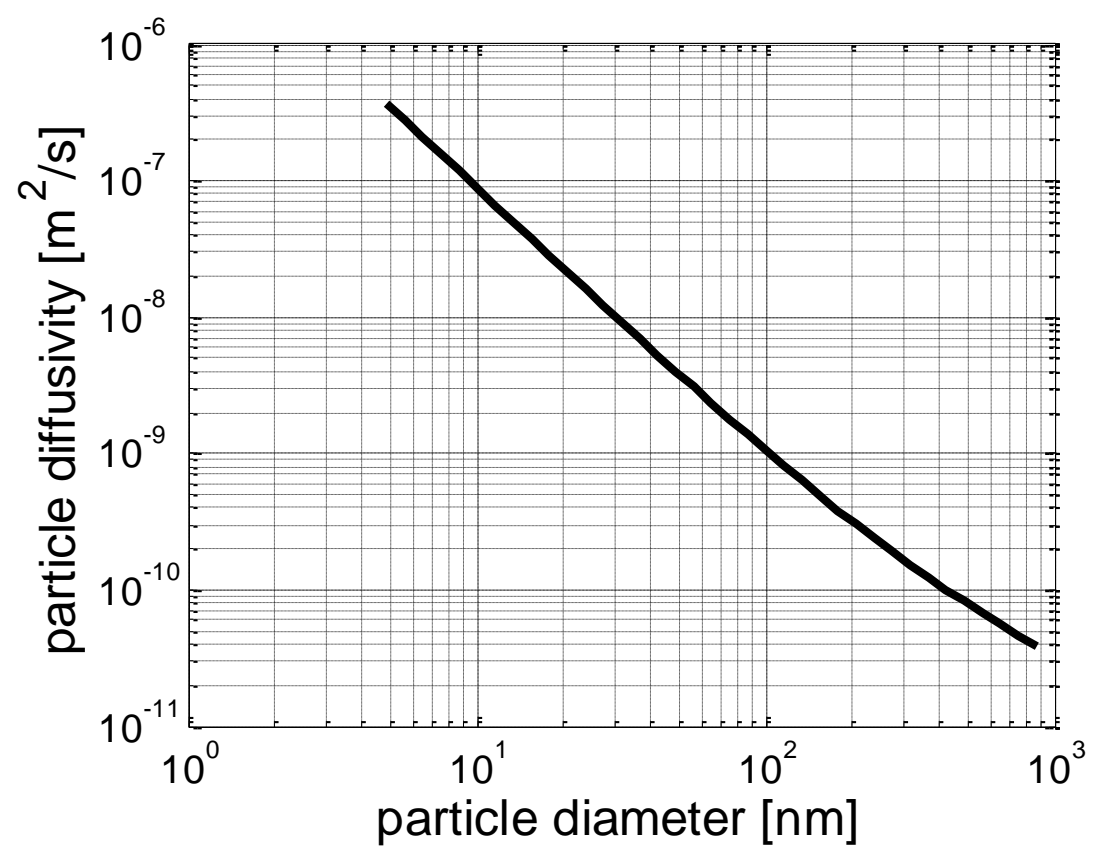

Figure 7: Particle diffusivity as a function of the particle size. 
To appreciate the respective contribution to the apparent capture efficiency from wall deposition (a diffusive process) and from complete evaporation, the time scales of the two processes are compared in Figure 8. Here, the time scale of evaporation is defined as the time needed to evaporate $99 \%$ of the particle mass of a pure HC particle (group III) according to eq. (2). It is evident that this time scale is significantly shorter than the time scale of diffusion, irrespective of whether the diffusion distance is taken to be the radius of the channel or the distance from a normalized radial position of 0.5 to the wall. Hence, it may be concluded that the effect of total evaporation dominates the effect of particle diffusion everywhere except at very short distances from the wall.

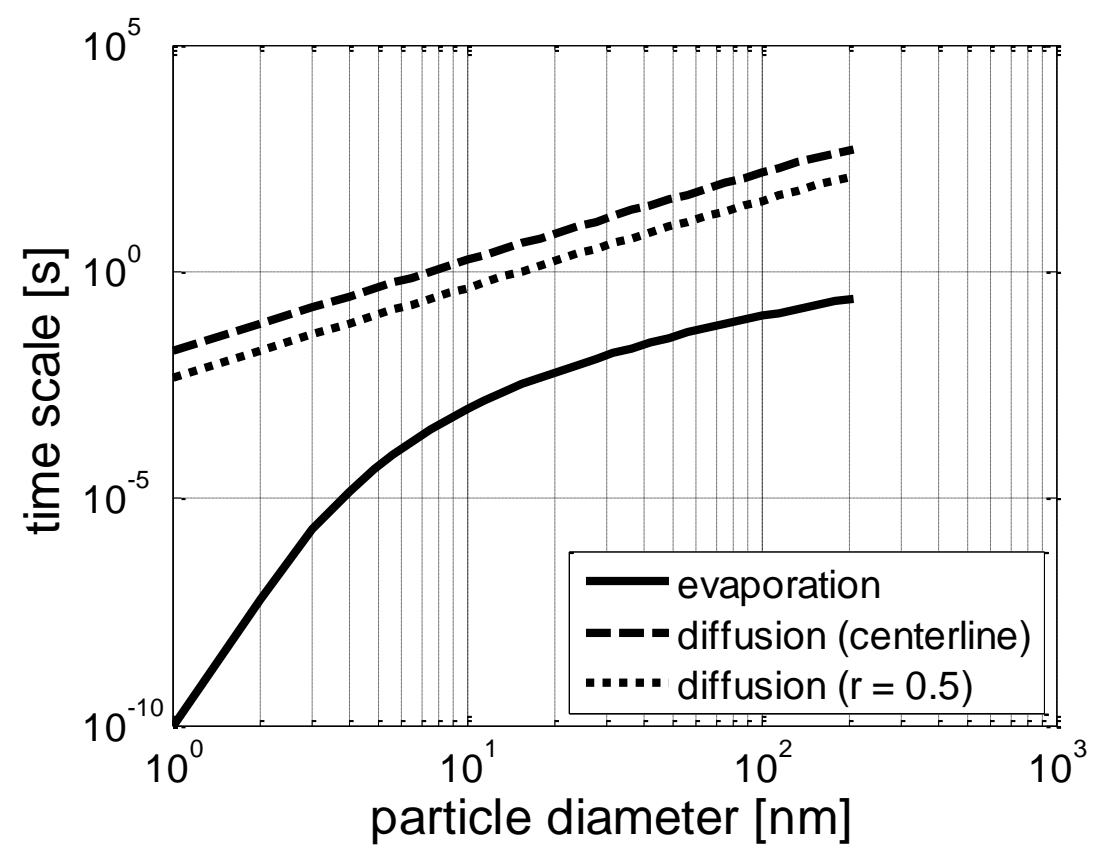

Figure 8: The time scales of particle diffusion to the wall in the validation simulation (from the centerline and from normalized radial position $r=0.5)$ and the time scale of HC evaporation.

\subsection{PM transformations under real engine conditions}

The conceptual model has been developed with the aid of experiments carried out at retention times significantly longer than those observed under typical real engine conditions. The background to this approach lies in the fact that the observed particle trapping efficiencies are typically very low in open substrates at real engine conditions, and that experimental data for the validation of numerical models therefore cannot be readily obtained. This fact does not however compromise the usefulness of the conceptual model in the development of comprehensive filter models or in the monitoring of experiments, but it does not directly reveal what the implications are of the PM transformations for the operation of a real monolithic reactor. To assess the influence of PM transformations under more realistic operating conditions, the validation simulation is therefore rerun at a linear gas velocity of 10 $\mathrm{m} / \mathrm{s}$ (corresponding to a space velocity of approximately $240,000 \mathrm{~h}^{-1}$ ). This increase in the gas linear velocity corresponds to an increase in the channel Reynolds number from 2 to 386.

Figure 9 illustrates the changes in the HC concentration fields due to the increase in gas velocity. The shorter retention time at higher velocity makes the adsorption of HCs in the washcoat less efficient, which leads to increased levels of $\mathrm{HC}$ in the gas bulk. An assumption of negligible background levels of HCs is clearly not appropriate at this high space velocity. Nevertheless, the reduction in gas phase $\mathrm{HC}$ levels throughout the channel at realistic engine conditions is still significant. As the HC 
evaporation rate is directly proportional to the HC partial pressure difference between the particle surface and the bulk, the reduction expected at the worst conditions (i.e. along the centerline) is of the order of $60 \%$. Given the very fast rate of $\mathrm{HC}$ evaporation at the current temperatures, one could therefore expect the shortened retention time for the particles in the channel to have a more pronounced effect on the rate of $\mathrm{HC}$ removal than the changes to the $\mathrm{HC}$ concentration fields, due to the non-linear dependence on the retention time (cf. eq (2)).

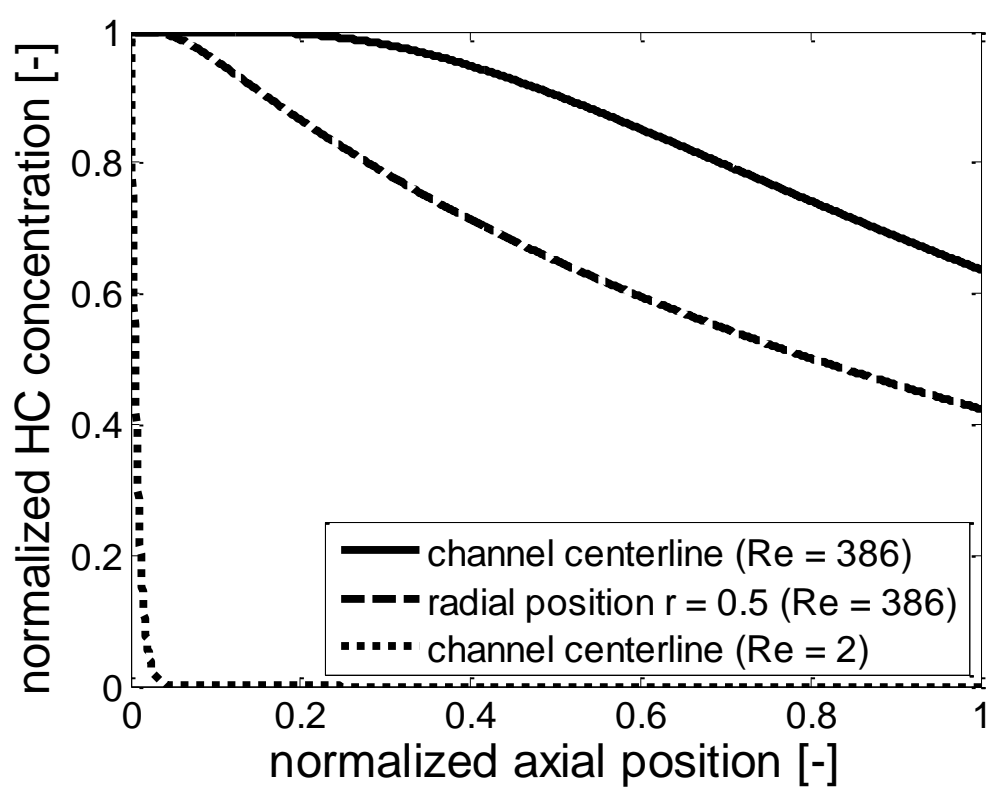

Figure 9: $H C$ concentration profiles in the validation simulation $(R e=2)$ and in the real engine simulation $(R e=386)$. For the real engine simulations, the HC profile is shown along two lines in the axial direction through the channel: one at the channel centerline and one midway between the centerline and the wall (radial position $r=0.5$ ). For the validation simulation, the two profiles along these lines almost overlap in linear scale, and only the centerline profile is shown.

In Figure 10, the apparent capture efficiency in the real engine simulation is plotted together with a corresponding case without $\mathrm{HC}$ evaporation.[21] It is clear that the two curves are similar for large particle sizes, whereas there is a marked increase in the apparent capture efficiency for smaller particles compared to wall deposition theory. Support for these findings can be found in Figure 8: Although the retention time in the monolith channel is short at realistic engine conditions, it is long enough to allow a significant $\mathrm{HC}$ evaporation from the smallest particles. However, the HC evaporation is no longer complete, as in the validation simulation. As can be seen from Figure 11, the overall number-based apparent capture efficiency of HC-containing particles at the channel outlet at these realistic engine conditions is $79 \%$. The present results thus suggest that the apparent capture efficiency may be higher than that predicted from deposition theory alone also at realistic engine conditions, but also that the $\mathrm{HC}$ stripping from the particles entering a monolith substrate at realistic conditions will not be complete. 


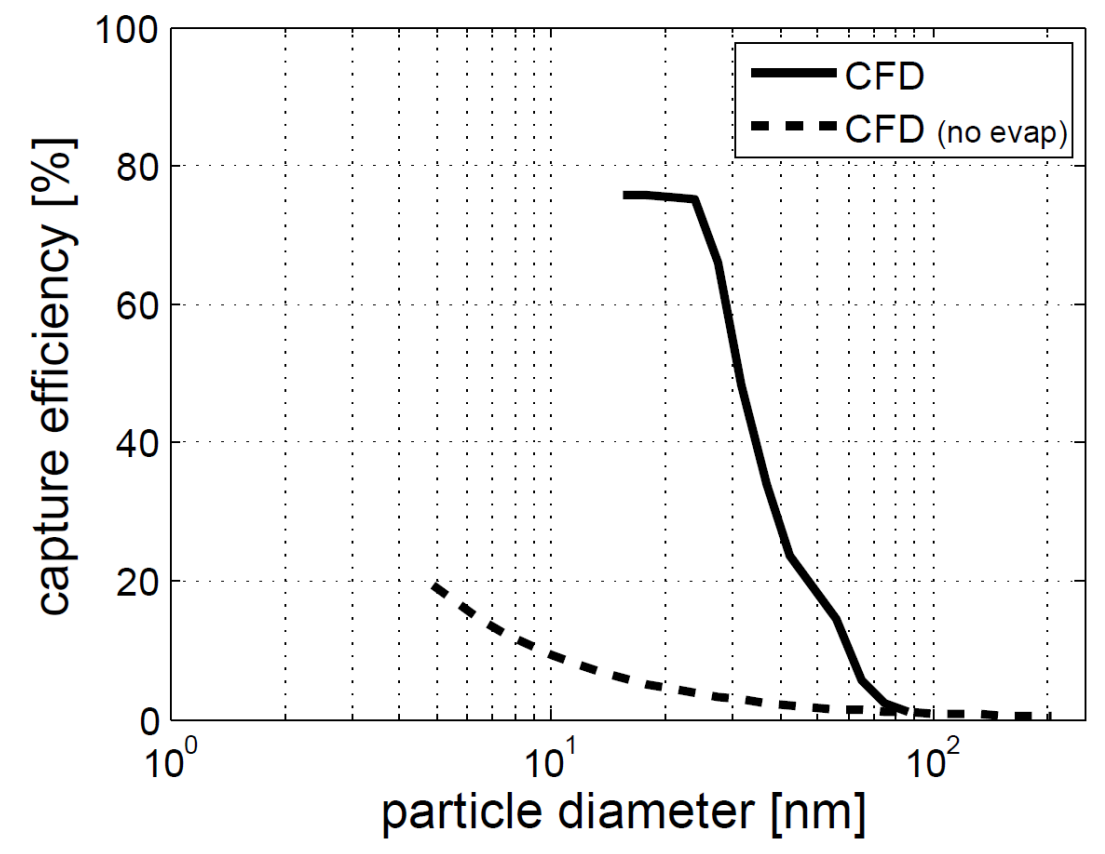

Figure 10: Apparent capture efficiency in the realistic simulation. Also plotted is the capture efficiency due to wall deposition only (no evaporation) of the untransformed injected PSD.

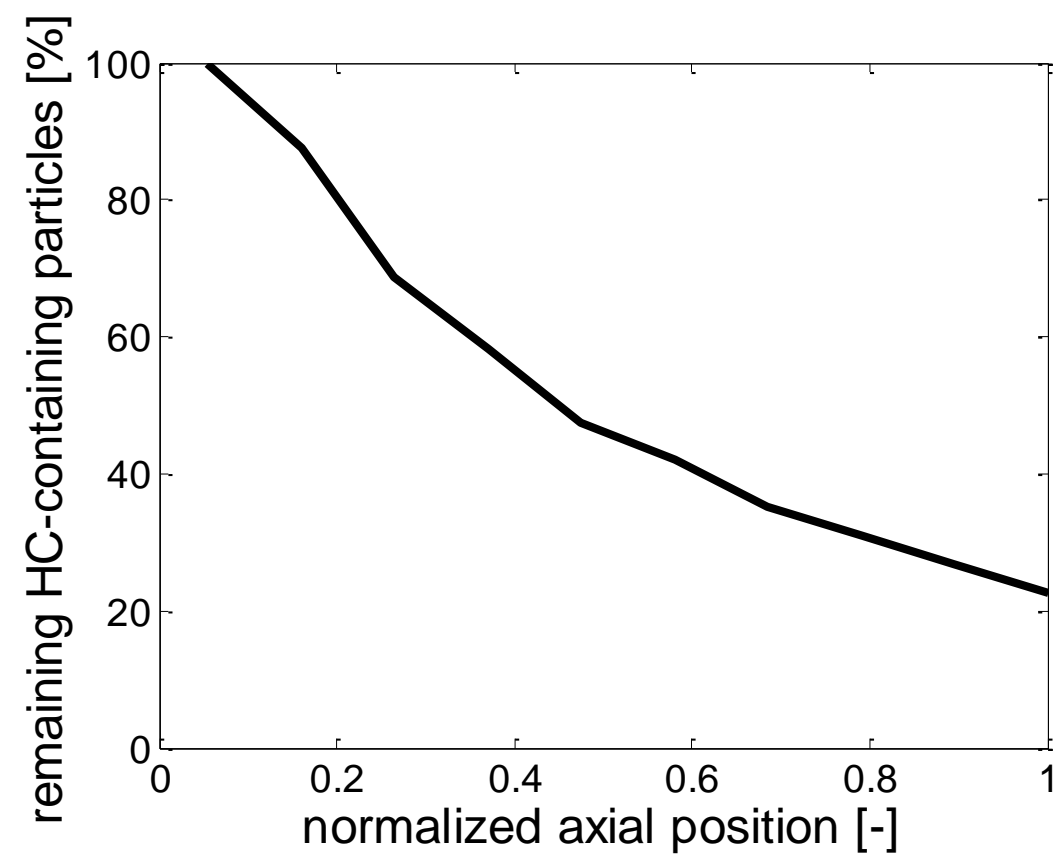

Figure 11: Number percentage of HC-containing particles present in the channel as a function of the axial distance into the monolith channel for the realistic simulation.

\subsection{Usefulness of the current methodology}

The important implications of this work are that the proposed conceptual model, in its tanks-in-series version, can be used in-situ for PM characterization in full scale experimentation rigs. In such a setup with, for example, a diesel oxidation catalyst (DOC) and a (wall-flow) diesel particulate filter (DPF), one could insert sampling probes before the DOC and between the DOC and the DPF. These probes would be connected via a switching valve to allow alternating sampling. The sample flow would 
finally be passed on to whatever gas analysis equipment that is used in the experiments (e.g. FTIR etc) at the volumetric flow rates typically used for such equipment (e.g. of the order of $8 \mathrm{slpm}$ ). In this sample line, one could then place an inert monolith whose volume is chosen in relation to the volumetric sample flow rate so as to allow a long enough retention time (the long retention time is needed here to allow complete HC evaporation). Finally, sample tubes connected to a PM analyzer (e.g. a DMS500) would be placed before and after this inert monolith, so that the CE can be determined and logged continuously. With this proposed setup, one would be able to sample the gas flow in relevant locations in the full scale experimentation rig and - by using the measured CE curve with the herein discussed conceptual model - obtain a simultaneous characterization of the PM flowing into the DOC, and out from the DOC into the DPF. Important data that would be acquired in this way include:

- the relative amounts of $\mathrm{HCs}$ in the PM currently existing in the exhaust pipe

- the degree of HC stripping in the DOC at the current operating conditions

- the particle size-resolved HC content of the PM currently being loaded into the DPF

In particular, the information about the PM entering the DPF would be highly significant for developers of PM filtration, accumulation and oxidation models for DPFs. In the development of more detailed and accurate chemical kinetics for the reactions of diesel and gasoline particulate matter in filters, it will be necessary to also be able to predict the time-resolved properties of the particles collected in the filter (i.e. reactivity, amount of adsorbed hydrocarbons, etc.). It is shown here that the data necessary to construct such models could possibly be obtained in-situ with the aid of the herein presented conceptual model and PM measurements over an inert open substrate.

It is worth pointing out here that certain assumptions in the conceptual model in its current implementation (e.g. the choice of tetracontane as the representative HC) can easily be relaxed or replaced by choices more relevant to a certain modeling campaign. There will always be a variety of HCs adsorbed on the PM, as well as a variety of PM shapes and reactivity. The representation of HC as tetracontane and the use of a sigmoid function to describe the relative amounts is at best a good representation of the reality. However, the usefulness becomes clear when analyzing the fitted HC content. As an example, in [21] a peak of small size in the PSD could be interpreted as volatiles, but in fact it was demonstrated that the peak was from non-volatile PM, possibly ash content when considering the engine operation. Finally, when used for full scale experimentation (as described above), the residence time and temperature in the inert monolith would need to be adjusted to be able to extract the necessary characteristics.

\section{Conclusions}

The current work presents and validates a conceptual model for transformations of particulate matter during its transport and deposition in a monolith channel. The model can be used either within a tanksin-series framework (e.g. for parameter fitting purposes) or within a finite-volume framework (e.g. for filter design purposes). It is shown that the model is able to explain the observed (apparent) changes to the particle size distribution for a particulate flow passing through a monolith channel, which could not be predicted by Brownian deposition theory alone.

It is shown in this work that, when the monolith channel acts as a $\mathrm{HC}$ sink, the $\mathrm{HC}$ concentration in the carrier gas decreases and this drives off HCs from the PM. However, if the retention time in the channel is short, the driving force might not be high enough to allow for complete evaporation of HCs (due to incomplete adsorption of HC onto the channel walls). Furthermore, a short retention time in itself acts so as to diminish the extent of PM transformations. It should however be stressed that PM transformation can be effective also at incomplete $\mathrm{HC}$ evaporation, since particles that shrink attain a higher diffusivity and thus may deposit more efficiently inside the channel.

In the development of more detailed and accurate chemical kinetics for the reactions of diesel and gasoline particulate matter in filters, it will be necessary to also be able to predict the time-resolved properties of the particles collected in the filter (i.e. reactivity, amount of adsorbed hydrocarbons, ash 
content, etc.). The important implication of this work is therefore that such models can be constructed with the aid of carefully gathered data from experiments on open substrates, where the open substrate may act as an in-situ PM analyzer in the full scale experimentation.

\section{Nomenclature}

Abbreviations

\begin{tabular}{ll}
\hline CE & capture efficiency \\
DOC & diesel oxidation catalyst \\
DPF & diesel particulate filter \\
HC & hydrocarbon \\
PE & penetration efficiency \\
PM & particulate matter \\
PSD & particle size distribution
\end{tabular}

Latin letters

\begin{tabular}{|c|c|}
\hline 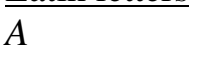 & $\mathrm{HC}_{\text {frad }} / \mathrm{HC}_{\text {part }}$ parameter [-] \\
\hline$B$ & $\mathrm{HC}_{\text {frad }} / \mathrm{HC}_{\text {part }}$ parameter [-] \\
\hline$a_{C}$ & evaporation coefficient $[-]$ \\
\hline$A_{p}$ & particle surface area $\left[\mathrm{m}^{2}\right]$ \\
\hline$A_{s}$ & monolith channel surface area $\left[\mathrm{m}^{2}\right]$ \\
\hline$c_{p}$ & particle heat capacity $[\mathrm{J} / \mathrm{kg}, \mathrm{K}]$ \\
\hline$C_{C}$ & Cunningham correction $[-]$ \\
\hline$d_{p}$ & particle diameter $[\mathrm{m}]$ \\
\hline$d_{c h}$ & monolith channel diameter $[\mathrm{m}]$ \\
\hline$D$ & diffusivity (of tetracontane) $\left[\mathrm{m}^{2} / \mathrm{s}\right]$ \\
\hline$D_{p}$ & particle (Brownian) diffusivity $\left[\mathrm{m}^{2} / \mathrm{s}\right]$ \\
\hline$h$ & heat transfer coefficient $\left[\mathrm{W} / \mathrm{m}^{2}, \mathrm{~K}\right]$ \\
\hline$h_{E}$ & sensible enthalpy $[\mathrm{J} / \mathrm{kg}]$ \\
\hline$h_{m}$ & average mass transfer coefficient $[\mathrm{m} / \mathrm{s}]$ \\
\hline$\Delta H_{\text {vap }}$ & heat of vaporization of tetracontane $[\mathrm{J} / \mathrm{kg}]$ \\
\hline $\mathrm{HC}_{\text {part }}$ & number fraction of pure $\mathrm{HC}$ particles $[-]$ \\
\hline $\mathrm{HC}_{\text {frac }}$ & mass fraction of HCs [-] \\
\hline$k_{B}$ & Boltzmann constant $[\mathrm{J} / \mathrm{kg}]$ \\
\hline$K$ & $\mathrm{HC}_{\text {frac }} / \mathrm{HC}_{\text {part }}$ parameter [-] \\
\hline$m_{H C}$ & mass of a tetracontane molecule $[\mathrm{kg}]$ \\
\hline$m_{p}$ & particle mass $[\mathrm{kg}]$ \\
\hline$M$ & $\mathrm{HC}_{\text {frac }} / \mathrm{HC}_{\text {part }}$ parameter [-] \\
\hline$M_{H C}$ & molar mass of tetracontane $[\mathrm{kg} / \mathrm{mol}]$ \\
\hline$N_{C}$ & total number of parcels in the cell [-] \\
\hline$N_{P}$ & number of real particles per computational parcel [-] \\
\hline$N u_{\infty, T}$ & asymptotic Nusselt number [-] \\
\hline$p_{H C, \infty}$ & partial pressure of tetracontane in the cell $[\mathrm{Pa}]$ \\
\hline$p_{H C, s}$ & partial pressure of tetracontane at the particle surface $[\mathrm{Pa}]$ \\
\hline $\begin{array}{l}p_{H C, s a t} \\
P\end{array}$ & $\begin{array}{l}\text { saturation vapour pressure of tetracontane at the current conditions }[\mathrm{Pa}] \\
\text { pressure }[\mathrm{Pa}]\end{array}$ \\
\hline$q$ & volumetric flow rate $\left[\mathrm{m}^{3} / \mathrm{s}\right]$ \\
\hline$Q$ & $\mathrm{HC}_{\text {frac }} / \mathrm{HC}_{\text {part }}$ parameter $[-]$ \\
\hline$r$ & normalized radial position [-] \\
\hline$r_{\mathrm{x}}$ & uniformly distributed random number on the interval $(0,1)[-]$ \\
\hline$R$ & universal gas constant $[\mathrm{J} / \mathrm{K}, \mathrm{mol}]$ \\
\hline$S_{H}$ & heat source term $\left[\mathrm{W} / \mathrm{m}^{3}\right]$ \\
\hline$S_{M}$ & mass source term $\left[\mathrm{kg} / \mathrm{m}^{3}, \mathrm{~s}\right]$ \\
\hline$S h$ & Sherwood number $[-]$ \\
\hline
\end{tabular}




$\begin{array}{ll}t & \mathrm{HC}_{\text {frac }} / \mathrm{HC}_{\text {part }} \text { parameter [-] } \\ T & \text { temperature }[\mathrm{K}] \\ T_{p} & \text { particle temperature }[\mathrm{K}] \\ T_{r e f} & \text { reference temperature }[\mathrm{K}] \\ u & \text { velocity }[\mathrm{m} / \mathrm{s}] \\ u_{p, i} & \text { particle velocity (in coordinate direction } i)[\mathrm{m} / \mathrm{s}] \\ v & \mathrm{HC}_{\text {frac }} / \mathrm{HC}_{\text {part }} \text { parameter }[-] \\ V_{C} & \text { volume of a computational cell }\left[\mathrm{m}^{3}\right] \\ x_{p, i} & \text { particle position (in coordinate direction } i)[\mathrm{m}] \\ Y_{H C} & \text { mass fraction of HC }[-] \\ z & \text { axial position in the monolith channel }[\mathrm{m}] \\ z^{*} & \text { dimensionless axial coordinate }[-]\end{array}$

\section{Greek letters}

$\gamma$

$\varsigma_{i}$

$\rho$

$\rho_{H C}$

surface tension of the tetracontane-air interface $[\mathrm{N} / \mathrm{m}]$

normally distributed random number of zero mean and unit variance [-]

density $\left[\mathrm{kg} / \mathrm{m}^{3}\right]$

$\mu$

density of tetracontane $\left[\mathrm{kg} / \mathrm{m}^{3}\right]$

dynamic viscosity $[\mathrm{Pa}, \mathrm{s}]$

\section{Acknowledgements}

The computations were partly performed on resources at Chalmers Centre for Computational Science and Engineering (C3SE) provided by the Swedish National Infrastructure for Computing (SNIC).

\section{References}

[1] W. C. Hinds, Aerosol Technology, 2nd ed, Wiley, 1998.

[2] European Environment Agency, Air pollution in Europe 1990-2004, 2007.

[3] A.G. Konstandopolus, M. Kostoglou, N. Vlachos, E. Kladopoulou, Adv. Chem. Eng. 2008, 33, 213.

[4] N. Zimmerman, K. J. Godri Pollitt, C.-H. Jeong, J. M. Wang, T. Jung, J. M. Cooper, J. S. Wallace, G. J. Evans, Atmos. Environ., In Press, doi:10.1016/j.atmosenv.2013.12.023, 2014.

[5] H. Burtscher, J. Aerosol Sci. 2005, 36, 896.

[6] B. Giechaskiel, A. Mamakos, J. Andersson, P. Dilara, G. Martini, W. Schindler, A. Bergmann, Aerosol Sci. Tech. 2012, 46, 719.

[7] S. Collura, N. Chaoui, B. Azambre, G. Finqueneisel, O. Heintz, A. Krzton, A. Koch, J. V. Weber, Carbon 2005, 43, 605.

[8] M. Maricq, R. E. Chase, D. H. Podsiadlik, R. Vogt, SAE Tech. Paper 1999, 01-1461.

[9] H. Sakurai, H. J. Tobias, K. Park, D. Zarling, K. S. Docherty, D. B. Kittelson, P. H. McMurry, P. J. Ziemann, Atmos. Environ. 2003, 37, 1199.

[10] A. Messerer, R. Niessner, U. Pöschl, Carbon 2006, 44, 307.

[11] H. Sakurai, K. Park, P. H. McMurry, D. D. Zarling, D. B. Kittelson, P. J. Ziemann, Environ. Sci. Technol. 2003, 37, 5487.

[12] J. Schneider, N. Hock, S. Weimer, S. Borrmann, U. Kirchner, R. Vogt, V. Scheer, Environ. Sci. Technol. 2005, 39, 6153.

[13] S. J. Harris, M. M. Maricq, Aerosol Sci. 2001, 32, 749.

[14] S. J. Harris, M. M. Maricq, J. Aerosol Sci. 2002, 33, 935.

[15] A. Zucca, D. L. Marchisio, A. A. Barresi, R. O. Fox, Chem. Eng. Sci. 2006, 61, 87.

[16] T. Rönkkö, A. Virtanen, J. Kannosto, J. Keskinen, M. Lappi, L. Pirjola, Environ. Sci. Technol. 2007, 41, 6384.

[17] E. Vouitsis, L. Ntziachristos, Z. Samaras, Atmos. Environ. 2005, 39, 1335. 
[18] K. Vaaraslahti, J. Ristimäki, A. Virtanen, J. Keskinen, B. Giechaskiel, A. Solla, Environ. Sci. Technol. 2006, 40, 4776.

[19] D. B. Kittelson, W. F. Watts, J. Johnson, Diesel Aerosol Sampling Methodology - CRC E-43: Final report, 2002.

[20] D. B. Kittelson, J. Aerosol Sci. 1998, 29, 575.

[21] J. Sjöblom, H. Ström, Ind. Eng. Chem. Res. 2013, 52, 8373.

[22] J. Sjöblom, Top. Catal. 2013, 56, 287.

[23] B. Giechaskiel, Y. Drossinos, SAE Int. J. Engines 2010, 3, 1140.

[24] J. E. Johnson, D. B. Kittelson, Appl. Catal. B 1996, 10, 117.

[25] E. Tronconi, P. Forzatti, AIChE J. 1992, 8, 201.

[26] F. J. Richards, J. Exp. Bot. 1959, 10, 290.

[27] H. Ström, S. Sasic, B. Andersson, B., Chem. Eng. J. 2010, 165, 934.

[28] H. Ström, B. Andersson, Top. Catal. 2009, 52, 2047.

[29] H. Mikami, Y. Endo, Y. Takashima, Int. J. Heat Mass Transfer 1966, 9, 1435.

[30] C. M. Rhie, W. L. Chow, AIAA J. 1983, 21, 1525.

[31] S. Chandrasekhar, S., Rev. Mod. Phys. 1943, 15, 1.

[32] E. Peirano, S. Chibbaro, J. Pozorski, J. P. Minier, Progr. Energy Comb. Sci. 2006, 32, 315. 